\title{
Toward a Hybrid Approach for Crowd Simulation
}

\author{
Chighoub Rabiaa \\ Department of Computer Sciences \\ Lesia Laboratory, Biskra University \\ Biskra, Algeria
}

\author{
Cherif Foudil \\ Department of Computer Sciences \\ Lesia Laboratory, Biskra University \\ Biskra, Algeria
}

\begin{abstract}
We address the problem of simulating pedestrian crowd behaviors in real time. To date, two approaches can be used in modeling and simulation of crowd behaviors, i.e. macroscopic and microscopic models. Microscopic simulation techniques can capture the accuracy simulation of individualistic pedestrian behavior while macroscopic simulations maximize the efficiency of simulation; neither of them assures the two goals at the same time. In order to achieve the strengths of the two classes of crowd modeling, we propose a hybrid architecture for defining the complex behaviors of crowd at two levels, the individual behaviors, and the aggregate motion of pedestrian flow. It consists of combining a microscopic and a macroscopic model in an unified framework, we simulate individual pedestrian behaviors in regions of low density by using a microscopic model, and we use a faster continuum model of pedestrian flow in the remainder regions of the simulation environment. We demonstrate the flexibility and scalability of our interactive hybrid simulation technique in a large environment. This technique demonstrates the applicability of hybrid techniques to the efficient simulation of large-scale flows with complex dynamics.
\end{abstract}

Keywords-crowd behavior; micro-scale representation; multilayered framework; real time simulation

\section{INTRODUCTION}

Simulating the movement of pedestrian crowd is a field of research that receives significantly growing interest during the last years, due to their vast domains of application: movies industry and entertainment, security, emergency training, architecture, and ma ny others [5, 37]. Several models for crowd simulation [23, 37] have been proposed and many efforts have been made to modulate intuitive navigation control and real time crowd behavior simulation, these models were classified into two categories: microscopic and macroscopic [25]. There are two conflicting requirements in crowd simulation.

The first major problem is that the most applications require a human crowd simulated in real time, with higher level of detail and an accurate realism of behaviors. Then, there is still a clear relationship between the accuracy realism of crowd behaviors and the computational costs of simulation. Satisfying these both constraints at the same time is particularly a challenge of great importance [21]. The majority of the previous models have a limited ability to response to the latter problem, they tend to focus on a single factor; there is no existing method that is able to reduce the computational cost while maintaining the high level detail of simulation. Almost all the existing models were agent-based (microscopic models). This approach describes the most natural way to simulate crowds as independent autonomous pedestrians interacting with each other; it usually handles local collision avoidance and global navigation for each person. However, these kinds of models have the drawback that when animating a large crowd, they are computationally intensive. Microscopic models give more accurate results only for smaller crowds to achieve real time simulation. On the contrary, the macroscopic models are usually created to realize a real time simulation for very large crowds; they follow the features of the flow as long as the overall crowd behavior seems realistic. These models offer a coarse-grained simulation result with higher execution efficiency which is due to the lack of concerns on individual issues [25].

Finally, modeling the movement and behavior of the virtual crowd remains a major challenge as highly dynamic complex systems [10], the crowd is a large group of pedestrians with non-uniform spatial distribution and heterogeneous behavior characteristics, and it exhibits often distinct characteristics, such as independent behaviors, selforganization, and pattern formation, due to interactions among the individuals. Previous works have suggested that human crowd dynamic can be modeled on many different scales [25, 10], from coherent aggregate behaviors of the crowd on the largest scales to the individual behaviors, interactions among individuals on the small-scale detail. Such multi-scale systems are computationally expensive for traditional simulation techniques to capture over the full range of scales [31].

To overcome these two conflicting goals, we assume a scalable simulation is required to handle at least several hundreds or even thousands of pedestrians, running in realtime, particularly with respect to the complexity of the environment and the realism of behaviors required by the crowd, we investigate to find a good balance between visual credibility of complex crowd behaviors and computational requirements, where the behaviors of human crowd can be viewed on a two different level of detail: from the chaotic, fluctuating interactions between individual objects on the finest scales, to the coherent aggregate flow of the pedestrian crowd on the largest scales.

Our solution consists to introduce a hybrid simulation architecture that combines the strengths of two classes of crowd modeling to achieve flexible, interactive, high-fidelity simulation on large environment. This architecture couples a microscopic model of individual navigation [27] with a novel continuum approach for the collective motion of pedestrians; it can apply to simulate the behaviors and movement patterns of extremely large crowds at near real-time rates on 
commodity hardware. Our approach is able to determine by itself the most suitable model of modeling for each region in the environment, regarding the simulation context, in real time and within a continuous environment. To do so, we first introduce the generic notions of dynamic change of representation, and we describe our method for handling the transfer of pedestrian between continuum and discrete simulation areas and discuss how the constituent simulation components are adapted to handle this transition.Then, we evaluate this approach experimentally along two criteria: the impact of our methodology on the computational resources, and an estimation of the dissimilarity between a full microscopic simulation and a simulation with our methodology. Finally we discuss the results obtained and propose enhancements for future works.

The remainder of this paper is organized as follows. First, the related works on the simulation behavior of virtual crowd are introduced to give the readers some background information. Then, section 3 describes our hybrid framework for simulating the movement of crowds. The macroscopic and behavioral models are introduced in section 4 and section 5 respectively. We describe a strategy that allows dealing with the interaction and the online-switching of simulation models for crowd behaviors studying. Section 7 demonstrates the simulation results in several common scenarios. Finally, the conclusion section outlines the directions for future work.

\section{RELATED WORK}

Crowd simulation has been the subject of studies of computer animation for a long time, there is a large amount of relevant work in the pedestrian dynamics, path planning and navigation [10]. In this section, we highlight some of recent models designed for simulating the real time navigation of large number of pedestrians in complex dynamic environments. Depending on the levels of simulation resolution, the existing models are largely classified into two extreme categories [25], i.e., continuum macroscopic models $[28,18]$, and individual-based microscopic models $[9,1]$.

The microscopic scale of modeling typically involves a detailed design and considers each pedestrian as an autonomous individual that has its characteristics and intentions, and interacts with other pedestrians. A microscopic model normally engages the local behaviors and emergent phenomena which are based on changes in pedestrian surrounding environment. These models include the social force model [30,9], cellular automaton model [1] and rule based model [7, 8].

A large body of other studies from a variety of scientific fields [37] has developed to simulate various aspects of crowd movements and dynamics. Musse and Thalmann [32] proposed a model for crowd simulation with hierarchically structured crowds having different levels of autonomy. Their model is based on groups, rather than individuals: groups' are more intelligent structures, where individuals follow the group's specification. A cognitive model of crowd behavior has been proposed [13] by applying the Festinger's (1954) social comparison theory, which is the general process underlying the social phenomena, to simulate pedestrian movement in a simple virtual environment. Pelechano et al.
[24] described a multi agent model called HiDAC model to simulate the flow of a high-density population in the dynamic virtual environment. It is further expanded by adding OCEAN personality model to demonstrate personality influences on the crowd motion [12]. Shao and Terzopoulos [35] used perceptual, behavioral, and cognitive models to simulate individuals.

The local navigation model of crowd alone cannot realistically simulate the behavior of pedestrians that try to reach some specified goals. Consequently various behavioral approaches have been combined with global navigation [30, 23, 36]. Sung et al. [19] proposed a model for developing situation-based crowd behavior based on combining probabilistic roadmaps with motion graphs to find paths and to compute detailed locomotion to steer characters to a goal. Guy et al. [34] proposed a new algorithm called "PLEdestrians" for simulating large crowds at interactive rates based on the Principle of Least Effort; this approach calculates a biomechanically energy-efficient, collision-free trajectory for each heterogeneous individual. In [16], a two level navigation method was introduced where the velocity obstacle concept from robotics is adapted to model human interactions. These geometric formulations are often based on (Reciprocal) Velocity Obstacles (RVO) and have been shown to exhibit many emergent crowd phenomena.

For simulating the realistic navigation in large and complex environments, a hierarchical representation is presented to handle the fast path planning [33]. Jiang et al. [14] proposed a semantic model for modeling multi-layered environment, where the semantic information is described by three different levels: a geometric level, a semantic level, and an application level. And each level contains different maps for different objectives. Lamarche and Donikian [11] presented a hierarchical path-planning and a reactive navigation algorithm based on topological pre-computation structure of the geometric environment. Pettré et al. [15] proposed a novel navigation approach which extracts automatically a navigation graphs from geometric model of an uneven and multilayer virtual scene and applied it to deal the path planning problem using a navigation graph. Sud et al. [4] present the Multi-agent Navigation Graph (MaNG) which is formed by the intersection of the regions of the first order Voronoi diagram with the second order Voronoi diagram. In [3, 29], Sud et al. suggested Adaptive Elastic Roadmaps (AERO), which are contain elastic edges that can modified along with the environment using particle-based dynamics simulators. Yersin et al. [6] present a hybrid approach for real time crowd motion planning. A navigation graph is used to divide the environment into zones of varying interest. The potential fields are used only for those parts of a navigation graph that lie in a high interest region. In the other regions, the pedestrian behaviors are ruled by the navigation graph and a short term avoidance algorithm.

A macroscopic level modeling considers an overall situation of a large crowd without taking into account local interactions; it is concerned with group behavior and deals with a crowd as a whole or fluid in the form of partial differential equations formulating the relationship between continuum density flow and average speed [28, 2]. Hughes 
[26] implemented a continuum approach inspired from fluid dynamics to simulate the two dimensional pedestrian flow, this approach was later extended by Treuille et al. [2], who presented the continuum model in order to generate realistic motion planning for crowds. Their method calculates the potential function for a group once, and then it simultaneously derives the optimal paths for all group members. Recently, Huang et al. [18] revisited a dynamic macroscopic model of pedestrian flow proposed by Hughes and advanced an efficient solution algorithm. Jiang et al. [14] used the continuum model to simulate plausible crowd movement within multi-floor structures, such as a large pedestrian overpass, a subway station and an office building.

Various works have proposed to combine different models together to build a hybrid model. Anh et al. [26] propose a hybrid approach that combines a macro and micro models to simulate the pedestrian movements in the study of evacuation problem on a road network. They use an agent based LeaderFollower approach to simulate the pedestrian behaviors in the cross-section (where a decision must be taken), and the movements in the straight parts of the streets are calculated by the LWR-model. A multi-resolution method is proposed in [20]; it consists to incorporate both complete macroscopic and microscopic models and executes them inter-changeably. In this approach, the macroscopic model can only be executed when crowd movement is mostly stable (or becomes stable eventually). In [22, 21], a hybrid modeling method is proposed for crowd simulation, which combines macroscopic and microscopic models in a single simulation and executes them simultaneously by applying them to multiple partitions of a corridor. However, global and individual issues cannot be simultaneously reflected for any simulation area. Current hybrid models have two common weaknesses. The key component of the hybrid simulation model is to define how the two different types of simulations are combined together; this consists to limit an arbitrary number of interface points on elected regions of the boundary (e.g., doors, cross-sections) where the pedestrians under one type of simulation must be transitioned into the other. Therefore, a transition is not possible on the whole border of a model. Another weak point of state-of the-art hybrid modeling is the inflexibility of dynamically-exchanging the combined models. There is no broad framework which supplies a method to adaptively change the pedestrian dynamic models in a region as needed to observe certain phenomena.

\section{OUR MODEL}

We propose a hybrid framework for real time simulation of pedestrian dynamics and movement patterns of huge crowd in a complex virtual environment. This solution preserves the granularity of simulation at the individual level to capture individualistic pedestrian behaviors, and at the same time is scalable and can richly exhibit emergent behaviors of dense crowds. The problem that we still try to address is both achieving realistic simulation and scaling up in terms of the number of individual that can plan their motion in real time. Our proposed architecture consists to integrate a more detailed approach with a coarser model, describing the individual pedestrian behaviors and crowd dynamics, within an unified crowd modeling framework, and execute them simultaneously in different regions of the virtual environment. There are several reasons which have motivated our decision of a proper coupling of two philosophies of modeling focusing on different level of detail (discrete individuals and crowd as a whole) executing to produce most visually pleasant simulation.

First, pedestrian crowd is a multi-scale phenomenon, which can be described at both macroscopic level (continuous medium) and microscopic level (granular medium), in many scenarios, it is need to have pedestrians behave individually, continuously interacting with other pedestrian while trying to reach their own objectives, thus the behaviors of each pedestrian must be treated more precisely. In other situation, pedestrians' flows demonstrate some striking similarities between pedestrians' behaviors and particle flow dynamics. Hence the flexibility of combining two models of different level of detail is examined to capture and characterize the almost aspects of the crowd dynamic. Macroscopic models allow a better overall understanding by regarding the crowd system as a whole rather than on the details, and are usually designed to achieve a coarse grained simulation executing in real-time for very large crowds as long as the overall crowd movement looks realistic. Microscopic models focus on individual behaviors including pedestrian's psychological and social characteristics, interaction among pedestrians, and complex cognitive behaviors. Although microscopic models are very accurate only for modeling smaller crowds to achieve real-time simulation, they can simulate pedestrian in a crowd with more realistic individual behaviors.

Secondly, a multi-methods simulation can give a good equilibrium between computing resources and simulation properties, such as realism, coherence and complexity. The microscopic models can generate a fine-grain simulation in more detail than the macroscopic method. However, they have high computational and memory needs. The macroscopic models can save resources but tend to give less accurate results. Mixing both types of models can hopefully allow combining the strengths of both classes of crowd modeling to achieve flexible, interactive, high-fidelity simulation on large virtual environment. However, this fundamental choice leads to several challenges which can be classified along two axes. The first key important of our hybrid technique is related to the execution of the models themselves. We must precise how the two different types of simulation will be used (the two models executes interchangeably or simultaneously), and the way they will coupled together. Finally, the second challenge focuses on the needs to manage the transfer of pedestrians between macroscopic and microscopic areas and we must discuss how the constituents of our hybrid framework are adapted to handle this transition. With the aim to achieve these two fundamental challenges, in our hybrid, multi-method technique we divide the simulation environment into multiple disjoint (and not necessarily connected) areas each area is ruled by either microscopic simulation or continuum simulation. These mutually exclusive regions are dynamic, we can adaptively change the simulation method used in a specific region as needed cording to its density, meet performance requirements, or to observe certain phenomena (individual behaviors or crowd movements). 


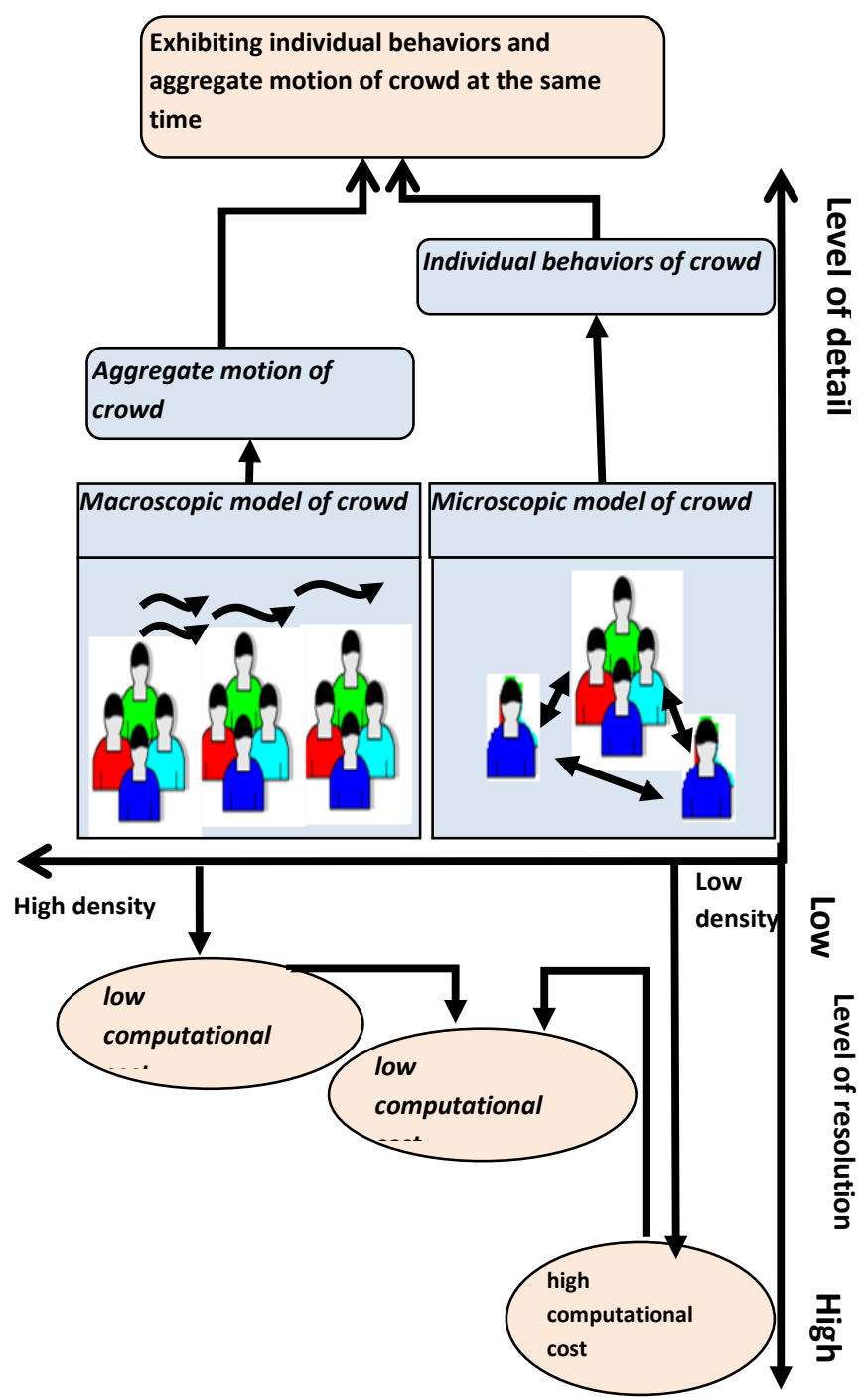

Fig. 1. Overview of hybrid model for real time crowd simulation

In the zones of high density of crowd the individual behavior is less critical, we assume to govern the crowd behaviors by a continuum approach to exhibit the aggregate motion and to describe behaviors similar to granular flows. On the other regions of low density, a realistic behavioral model is used to microscopically model the pedestrians, in these zones the standard representation of a crowd as a large group of pedestrian is used and individual behaviors is particularly relevant to the modeling process.

A challenging goal is to model these interactions in large crowd simulation systems at interactive rates. The areas of low density are exploited by a more realistic and accurate but expensive model, while computation time saved by using simpler and faster modeling approach in other regions. Our architecture also ensures that no visible disturbance is generated when switching from one model to another. To achieve this, we must be able to convert discrete pedestrian from microscopic simulation regions into the aggregate format necessary for continuum simulation (or vice versa).

\section{ENVIRONMENT MODEL}

The environment in which the simulation takes place is the surrounding of the pedestrians, where they move along, interact and navigate to get from one location to another, typically, it includes walk-able areas, obstacles of different natures, and destination. Whereas, fixed obstacles can be defined as regions that no pedestrian can access, moving obstacles are other pedestrians occupying predefined space from the environment which is consequently not anymore available [34].

The first step in designing a crowd navigation system is to construct an efficient abstract representation of the virtual environment where the pedestrian can rapidly perform wayfinding. We define a representation method which handles two types of structure data to clearly represent and to organize the topological relationship among the different geometrical areas of a large complex environment. This approach provides a well consistence resulting from the continuous interaction between two models of different level of detail.

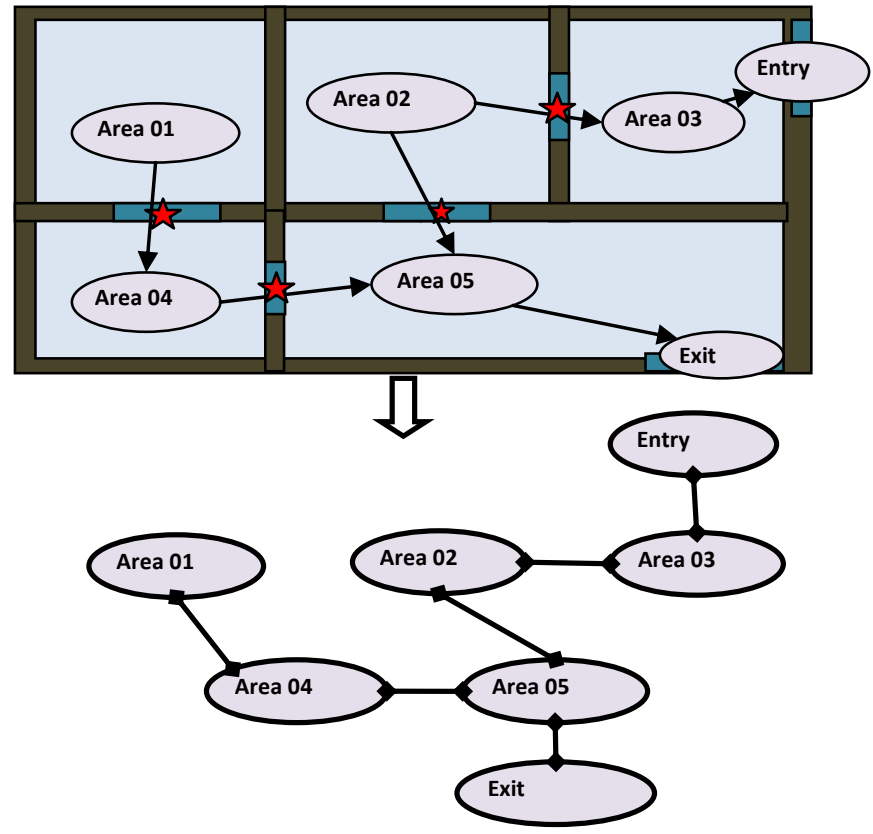

Fig. 2. Topological graph representation of the virtual environment

\section{A. Topological graph}

Usually, the virtual environment is defined by a 3D model to constitute a geometric representation of the real world. Such representation of the spatial data makes it difficult to handle by virtual pedestrian in order to find its own paths through the environment. The most way to facilitate this representation is to obtain the topological relation of the environment and its geographical areas captured in a graph based structure (Fig.2).

The topological graph uses nodes and edges to indicate the adjacency, connectivity, the inclusion and the intersection between the different parts of the environment, in which the node defines spatial areas and the possible path can be defined as edge. The internal spatial areas can be defined as a bounded volume in 3D space (such as a room, a corridor, a flight of 
stairs or even an entire floor) with bottom flat that contains several objects inside it (e.g., ground, walls, benches).

\section{B. Layered model for environment representation}

The second form of space representation is to use the layer structure (Fig (3)). We identified three independent layers related to the model used for simulating the behavior of virtual crowd, each of which contains both static and dynamic data. Then we implemented the following layers for representing each spatial area in the environment:

- Regional layer. In this level, the whole walking space of one spatial area is divided into a number of unique continuous sub-areas. For generating these sub-areas, one main requirement must be valid which is: these subareas must be exhaustive; two different must not cross each other. This level is used to precise which model must be used for movement modeling, macroscopic model in the subarea with high density, and the microscopic in the other sub-regions.

- Coarser layer. The surface of sub-area, defined in the regional level, is assumed to be divided into cells; we do not limit ourselves to a maximum of one pedestrian per cell, in contrast, we consider each cell's size to be sufficiently large enough to contain at least 25 individuals of average size, where individuals placed on the same cell do not overlap.

- Finer layer. Every cell from the coarser layer is further divided into a uniform lattice of cells, each representing a portion of the simulated environment and comprising information about its current state, both in terms of physical occupation by an obstacle or by a pedestrian. The size of the subareas could be reduced to the average space occupied by a single pedestrian.

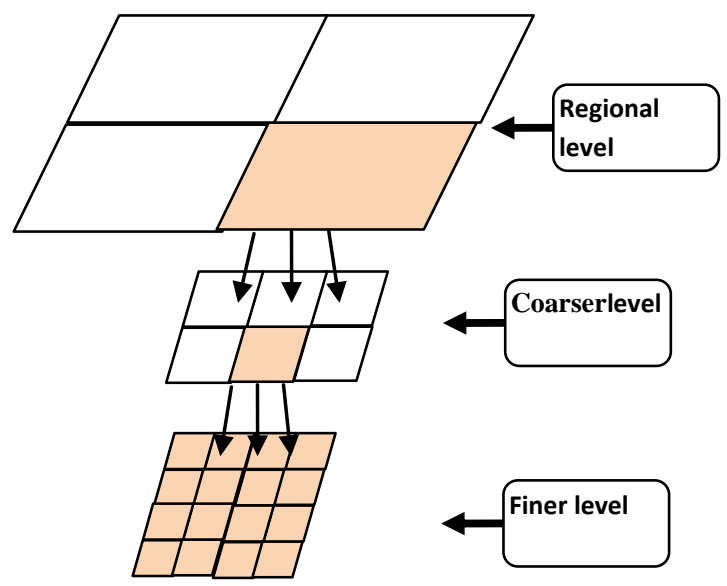

Fig. 3. Hierarchy model for environment representation

\section{MACROSCOPIC MODEL}

Macroscopic models deal with the whole crowd; they often use an analogy with fluid or gas dynamics to describe the evolution of crowd density and velocity in time by using partial differential equations [28, 18]. These models can be of value in simulating large-scale crowds and highly concentrated populations in spots such as stadiums, shopping malls, and subways. In the following sub-section, the development of the continuum model for modeling the pedestrian behavior problem is discussed.

Throughout the development of this continuum approach of simulation, different equations have been proposed to model the pedestrian movement in order to use the continuum model as a tool of analysis.

We refer that pedestrians waking in a $2 \mathrm{D}$ continuous surrounding environment are capable to find a specific path describing by its minimal travelling cost to achieve the goal. This can be express by an eikonal type equation [38].

\section{A. Governing equation}

Similar to other physical systems, the conservation law appears to be more effective when it charged to describe the crowd dynamics by evaluating the velocity, density and flow of pedestrian. The conservation of mass for pedestrian flows is defined as follow [39]:

$$
\begin{array}{r}
\rho_{t}(x, y, t)+\nabla \cdot F(\rho, x, y, t)=0, \quad \forall(x, y) \in \Omega, \mathrm{t} \in \mathrm{T} \\
\rho(x, y, 0)=\rho_{0}(x, y), \quad \forall(x, y) \in \Omega \quad(2)
\end{array}
$$

Where $\rho(\mathrm{x}, \mathrm{y}, \mathrm{t})$ (ped $\left./ \mathrm{m}^{2}\right)$ is the time-varying pedestrian density; $\Omega$ denotes a $2 \mathrm{D}$ continuous walking facility; $\mathrm{T}$ is the time horizon of analysis.

$$
F(\rho, x, y, t)=\left(f_{1}(\rho, x, y, t), f_{2}(\rho, x, y, t)\right)\left(\operatorname{ped} /\left(m \cdot s^{-1}\right)\right)
$$

is the vector of the flux in the walking facility and $f_{1}, f_{2}$ represent the flow in $\mathrm{x}$ - and $\mathrm{y}$-directions, respectively; $\|F(\rho, x, y, t)\|=\rho(x, y, t) U(\rho, x, y, t)$, where $U(\rho, x, y, t)(m /$ s) denotes the average walking speed of pedestrians at location $(x, y)$ at time $t$ which depend on the density and the free speed of pedestrian movement. Flow intensity or the flow-density relationship, $\|\mathrm{F}(\rho, \mathrm{x}, \mathrm{y}, \mathrm{t})\|$, is defined as

$$
\|F(\rho, x, y, t)\|=\sqrt{f_{1}^{2}(\rho, x, y, t)+f_{2}^{2}(\rho, x, y, t)}
$$

\section{B. Desired direction of crowd motion}

It is assumed that a pedestrian flow at location $(x, y)$ ? ? $\in$ $\Omega$ prefers to reach its goal as fast as possible, which implies that it selects the shortest path to move along. This path minimizes its individual cost potential to the destination, based on the instantaneous information that is available at the time decision making [40].

$$
C(\rho, x, y, t)=\frac{F(\rho, x, y, t)}{\|F(\rho, x, y, t)\|}+\nabla \Phi(x, y, t)=0
$$

Let $\Phi(\mathrm{x}, \mathrm{y}, \mathrm{t})$ be cost potential which is used to describe the minimal travel cost of pedestrians from location $(\mathrm{x}, \mathrm{y})$ ? $\in \Omega$ to the destination, i.e. it consists to record a value for each cell in the simulation environment where $(\mathrm{x}, \mathrm{y})$ ? ? $\mathrm{?}$ is the coordinate of the cell location. The value is assigned to 0 and positive infinity for cells in part of a goal exit and obstacle, respectively. Values for the other cells are assigned as positive values, which are determined during the model execution. $\Phi(\mathrm{x}, \mathrm{y}, \mathrm{t})$ is defined by the following Eikonal-type equation:

$$
\begin{gathered}
\|\nabla \Phi(\mathrm{x}, \mathrm{y}, \mathrm{t})\|=\mathrm{C}(\rho, \mathrm{x}, \mathrm{y}, \mathrm{t}) \text { in } \Omega \\
\Phi=0
\end{gathered}
$$


$C(\rho, x, y, t)(s / m)$ is the generalized local cost function which is assumed to depend on the local operating conditions in the walking facilities, i.e., the tendency to avoid highdensity regions. It takes the specific form $[38,39]$ :

$$
C(\rho, x, y, t)=\frac{1}{U(\rho, x, y, t)}
$$

\section{Crowd speed selection}

It is necessary to characterize how the magnitude of crowd velocity, i.e., the speed of crowd movement, changes with the crowd density. An exponential form of speed-density relationship is applied in the macroscopic model, as shown:

$$
\mathrm{v}(\rho)=\mathrm{v}_{\max } \mathrm{e}^{-\alpha\left(\frac{\rho}{\rho_{\max }}\right)^{2}}
$$

where $V_{\max }$ is a free flow speed, $\rho_{\max }$ is a congestion density [39].

\section{MicROSCOPIC SOLUTION FOR CROWD SIMULATION}

Our fundamental objective is to propose a behavioral approach, belonging to the microscopic philosophy, lies to reproduce the realistic navigation behaviors and the motion planning of crowd, so it also take into account the individual's detailed characteristics and behaviors of virtual pedestrian to provide a real time execution of coherent and interesting complex behaviors resulting from the evolution of system. This proposed solution should be able to simulate a large number of virtual pedestrian that behave like a large group of humans in the real world.

Obviously, the pedestrians are the strongly part of the simulation. A virtual pedestrian is defined as a fully autonomous entity which moves in a virtual environment with its own goals to achieve. It should look and behave in its own specific style as a real human in the life world, and be able to interact with the environment and its inhabitants in a seemingly intelligent way.

Our initial assumption is that the virtual pedestrian needs to be familiar with its surrounding because it has a mental map of the areas to be navigated and has a specific personality. During the navigation process, the pedestrian finds the shortest path and plans its movements to reach as specific destination as quickly as possible while avoiding bumping into other people, or tripping on an obstacle. As the pedestrian navigates, it has the capabilities of perceiving the virtual environment around them, analyzing environmental situations, and is exhibited natural behaviors. They should exhibit complex motions in large and dynamic environments. This process is repeated utile it reaches the goal position. We will address all these aspects of a pedestrian to look natural.

Our solution to tackle all these issues consists to design a layered framework that is scalable and efficient to target the real time simulation for low to medium scale crowd of people, where each person corresponds to a simulated pedestrian endowed with its own individual behavior. Particularly, this layered architecture is also easily expandable to incorporate more and more broad range complex and realistic behaviors at will, by simply integrating one or more new layers. Figure 4 shows the conceptual design of the framework. The framework consists of three levels, namely Physical layer, Behavioral layer, and Navigational layer. Every level contains a simple and/or complex highly independent task that may be executed by a pedestrian and has its own characteristics. This task will process inputs from the environment and produce as output an action for the pedestrian. Each level can be divided into modules which is associated in turn with more detailed and specific routines.

\section{A. Physical Layer}

The physical layer is the bottom level, it is comprises the perception and the action sub-modules.

- Perception module: is one which is responsible for collecting and acquiring sensory data by interpreting the virtual environment, it provides the virtual agent information about its surrounding space and makes it aware of the current situations for discuss the appropriate actions. In the simplest way, with this component, the pedestrian determines dynamically the current position, velocity and orientation of their neighbouring agents and the other visible elements depending on the agent's direction and field of view.

- Action module: This component including a walking sequence of animation which runs to achieve the locomotive tasks of pedestrians, then it transfers the behaviour determined by the behavioural level to the specific executions of the agent's effectors in the virtual space in order to move smoothly the pedestrian along the path that has been planned [27].

\section{B. Behavioral Layer}

Once the shortest path is computed by the navigational level, the next challenge is how the pedestrians follow, move and how evade each other naturally to achieve their goals. The proposed model deals with this problem by the behavioral level. The main role of this level is to address the realistic local movement of the individual pedestrians of the virtual crowd while preserving visually pleasing overall crowd behaviors. This is a challenging task, particularly in crowded scenarios with several hundred or thousands of entities where each one requires independently navigating in a decentralized way.

The behavioral layer uses [27] a microscopic approach of crowd simulation where the pedestrians are represented by a position in space, an orientation and a velocity. During each simulation phase, this level receives the information perceived by pedestrian from the physical level, and a set of way points representing the path calculated by navigational layer, and using it to decide the new behavior which is represented by determining the new location of pedestrian by modifying his orientation and/or his speed. This layer receives the information perceived by pedestrian from the physical level, and a set of way points representing the path calculated by navigational layer, and combines a forces model with behavioral rules to decide the new behavior which is represented by determining the new location of pedestrian by modifying his orientation and/or his speed. Finally this information is then sent to physical level in order to implement the walking animation. 


\section{Navigational Layer}

The Navigational layer is responsible to represent the navigation process. Hence, the navigation process will allow to people to follow the known route or choose a new path based on changes in the environment and their psychological parameters. This process focuses to analyze the representation and to compute an optimal path between any two locations in the environment. In this context, navigation process could be either local or global [27].

- Local Path Planning means the path is done while the pedestrian is moving; this process is capable of producing a new route in response to environment changes, i.e. user inserts or removes one or set of obstacles in the environment, or a door appearing blocked which makes that path invalid or creates a bottleneck in some part of the desired path. Specifically, the Local Path Planning Process is used in the case where the entity and its sub-goal are in the same region.

- Global Path Planning requires that the environment be completely known. In this approach, an offline process (before the simulation is started) generates a complete path from the start point to the destination point, and then the route is pre-calculated and stored in the topological graph in order to achieve real-time interactive navigation. The Global Path Planning Process is executed if the pedestrian and his sub-goal are in two different zones.

Both these two processes send the shortest path in form a set of waypoints (attractor points) to the Behavior Mechanism to carry out the required motion to reach it.

\section{INTERACTION BETWEEN MODELS}

Our approach proposed here for the design a hybrid crowd simulation model concentrates on the integration of two models that have different level of resolution in order to capture at the same time the micro and macro dynamics of human crowds. This type of simulation has the ability to divide the environment into multiple disjoint areas and to simultaneously execute these two models in different regions, by using a macroscopic modeling approach to simulate pedestrian flow in region of high density and a detailed microscopic model to simulate individual behaviors of pedestrian in the other regions. Investigating this integration makes clear two hard problems to be encountered:

First, assuring the consistency between the models is important for maintaining semantic continuity of results in terms of space (discrete/continuous), behaviors, and time, when concurrent interactions occur, if pedestrians pass from microscopic scale to macroscopic scale and vice-versa. Final issue consists to provide an efficient strategy for changing adaptively the simulation method in a specific region. It needs to identify the conditions in which a suitable modeling approach can be selected.

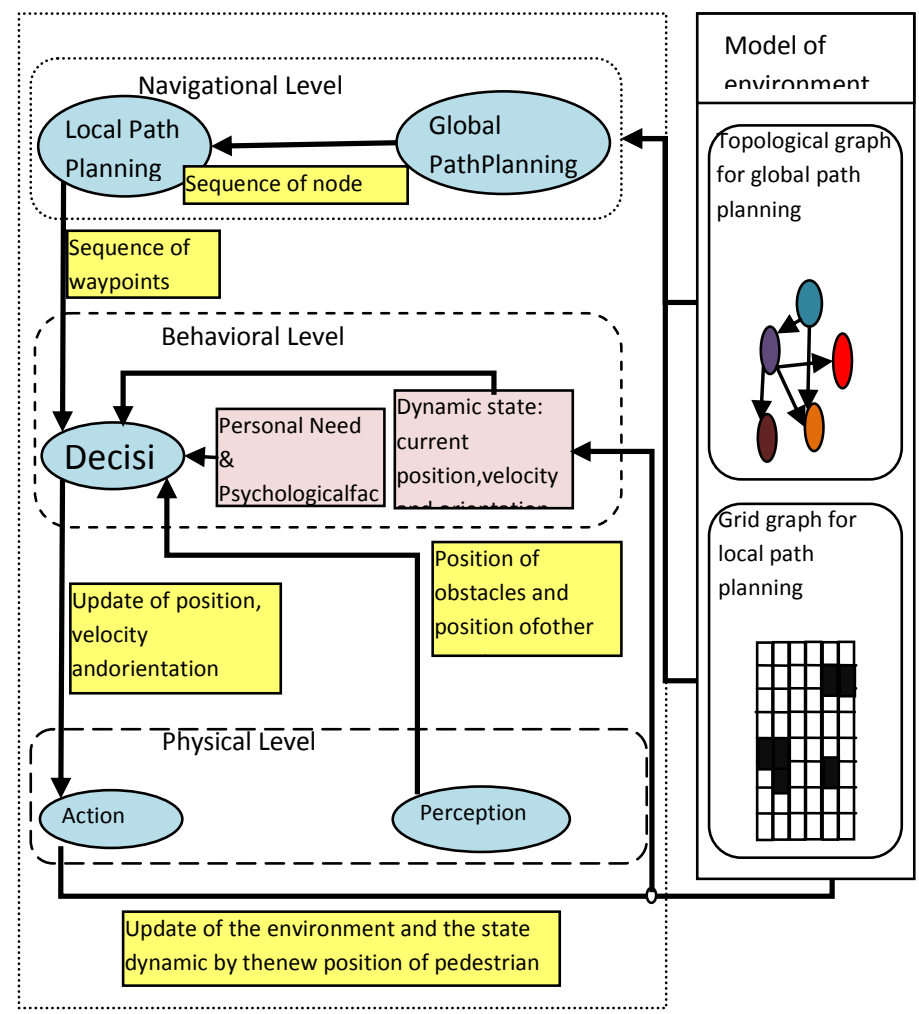

Fig. 4. Multi-layer architecture for modeling pedestrian movement, three levels are interesting physical layer, behavioral layer and navigational layer

\section{A. Transition of pedestrians}

As mentioned above, inconsistencies can arise in our hybridization solution when persons can be transformed from one model to another of different scale. To achieve this, an interface translating boundary condition is needed to define for moving those pedestrians from a continuous to a discrete modeling approach. We adapt this case by defining a boundary area adjacent to the macroscopic region; this bound is divided to cells, only the pedestrians in this area transform to the microscopic scale, i.e. if there are when pedestrians enter this area forcibly changing their representation levels, their positions, orientation and velocity will updated by the microscopic model.

There exist two basic communication operations between the two models: aggregation and disaggregation. The disaggregation refers to the process of generating the initial parameters for the microscopic model based on the result from the macroscopic model. Correspondingly, aggregation is the operation where the collects statistics from microscopic model and the parameters are generated in the format as required by the macroscopic model.

\section{B. Trigger}

Our hybrid approach of modeling consists to combine two models of different level of detail of simulation in a single framework; it attempts to provide a scalable and accurate method for the autonomous navigation process. 
The whole continuous simulation environment is divided into multiple mutually regions, defined at initialization. Each region is governed by one of two different motion modeling approaches, either macroscopic model or microscopic model.

According to the density of crowd, the model used in a specific region is determined at initialization and modifiable at runtime. Then it is very important to present an efficient technique to define how the simulation changes dynamically from one model to another. Density is another fundamental component of pedestrian flow models. As the density of pedestrians' increases, pedestrians will have less space to overtake other slow pedestrians and eventually the average walking speed is slowed down. Usually when the pedestrian density is higher than 5 6persons/m2, the average walking speed is so low that the crowd can hardly move any more.

- Switching Micro $\rightarrow$ Macro we calculate the density in each area. This operation occurs when the density in an area ruled by microscopic simulation is larger than a predefined threshold, then the system should trigger the execution of macroscopic model in this region.

- Switching Macro $\rightarrow$ Micro we calculate the density in each area, It consists to switch the simulation in a specific zone from a macroscopic to microscopic model; this is occurred when the density of this region is smaller than a threshold. Then the microscopic should execute.

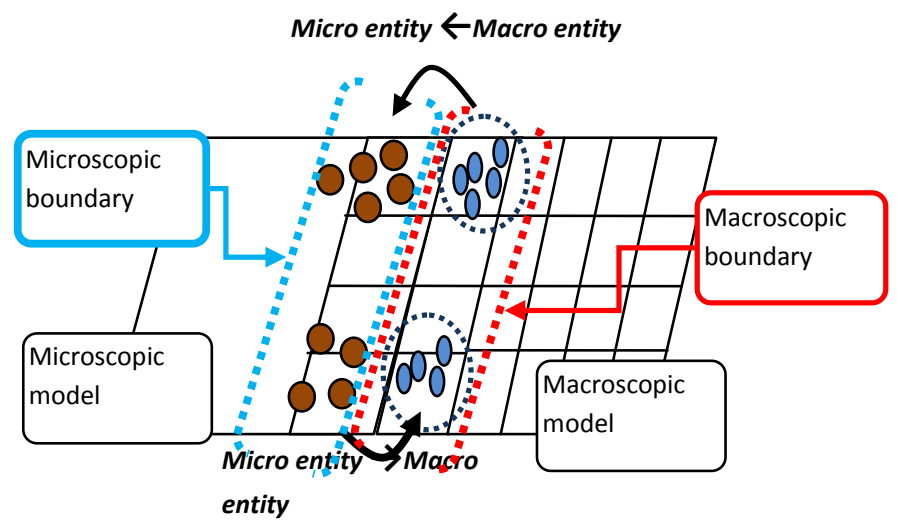

Fig. 5. Interaction between micro and macroscopic model

\section{RESULTS}

In this section we present and describe the experiments for the evaluation of our hybrid model for interactive visual simulation of large scale crowd of virtual pedestrian. Our solution model is specifically designed to support robust real time simulation of scenarios with thousands or even hundreds of thousands of pedestrians. It involves the combination of two significantly different types of modeling methodologies for taking the advantage of their complimentary features, in which a macroscopic model is applied where needed and a microscopic model where plausible.

In order to evaluate the effectiveness and the robustness of our multi-modeling approach presented in this paper, we have conducted to realize a number of simulations with different initial distributions and conditions (mainly changing the density of pedestrian crowd in the environment) in a situation in which experiments focused at analyzing the impact of the density of crowd on the pedestrian behavior that could be handled was being investigated.

The objective of the experiments which we use is to show the proposed model performs well to produce results that closely simulate real human behaviors in these situations, and to study whether the proposed model can describe the qualitative dynamic properties of the pedestrian's movement under situations with three different level of density (low, medium and high density) in terms of number of pedestrians that could be handled with reasonable performance.

We tested our system on virtual complex environment, in order to produce realistic crowd behaviors in this type of space; the simulation environment itself should have the features (properties) of real life environment. We also believe the representation of the environment has an important influence on pedestrian navigation. In this section we demonstrate the application of the hybrid approach using the arbitrarily complex geometry. The structure has a free floor space area comprising of 7 irregular shaped rooms with two external exits. The pedestrians are initially distributed randomly over the area of the environment. The particular distribution of the density pedestrian was selected to ensure that during the simulation all two possible modeling approaches: Macroscopic model; and microscopic model; would be used. We performed a series of experiments in order to test the behaviors under study focusing on showing the results of the interaction of the two sub-models.

Fig.6 shows the simulation results of the pedestrian dynamic produced by the microscopic model which is selected to apply according to the pedestrian density calculated as the numbers of pedestrians existing in the restricted areas under consideration. This model is considered to be qualitatively more accurate than the macroscopic model. This experiment shows that when the number of virtual pedestrian is small (Fig.6 (a)), the microscopic modeling approach has been employed to simulate each pedestrian as an individually entity with its own its own personality, and its behavior which is determined by both the global and local movement. In this scenario, we demonstrated that a leader has a major influence on people especially in evacuation situation, in order to formulate the leader-follow behaviors. Fig.6 (b) shows this case, when, we can observed the red flow follow a leader which has a global view of the simulated environment, then he find the shortest path into the exit, but the blue flow has no leader, then he choose the shortest door which leads it to follow the longest path (Fig. 6 (c, d)).

During the second experiment (Fig.7), we noticed that pedestrian's density increases in the same subarea; the macroscopic is adopted to handle the pedestrian's behaviors within a crowd of high density. This model facilitates the construction of small groups of individuals that shows a slight cohesion and natural fragmentation into subgroups that might be simple and therefore much more compact.

Group phenomenon is an interesting area of research for pedestrian simulation, because it is very common in the every- 
day life, people standing closer to its familiars and forming small groups. In panic situation, people relatively tend to gather together closer. In these situations, people are mostly linked by the (temporary) sharing of a common goal, and the overall group tends to maintain only week compactness, with a following behavior between members [27]. In Fig. 6, the macroscopic used to cluster the pedestrians into a structured group by assuming a common goal, passing a direction and speed that applied to all of the members. As a member of a group, each pedestrian coordinates with others in the same group and show an aggregate motion as they move together.

The last simulation results in fig (8) show that in crowded situations, (pedestrian's density increases until it reaches a maximum value when situation becomes congested), one of the typical phenomena occurring in pedestrian flow is selforganization of lane phenomena. In the real life, pedestrians in a crowded area tend to self-organize into lanes in order to reach their destination faster and easier.

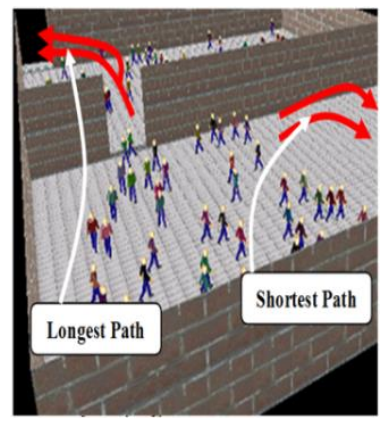

(a)

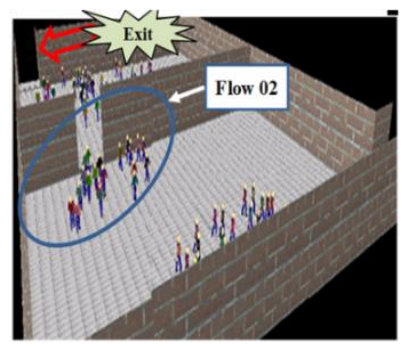

(c)

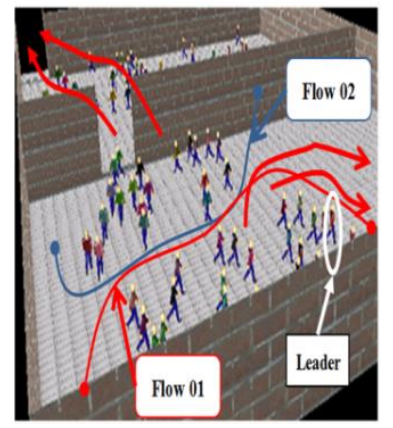

(b)

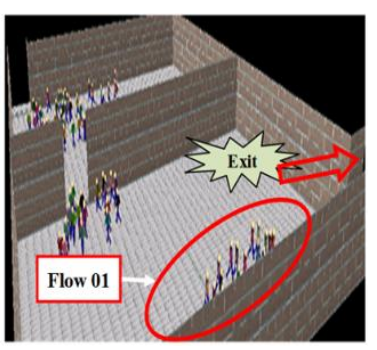

(d)
Fig. 6. The leader fined the shortest path, and the flow 1 follows it

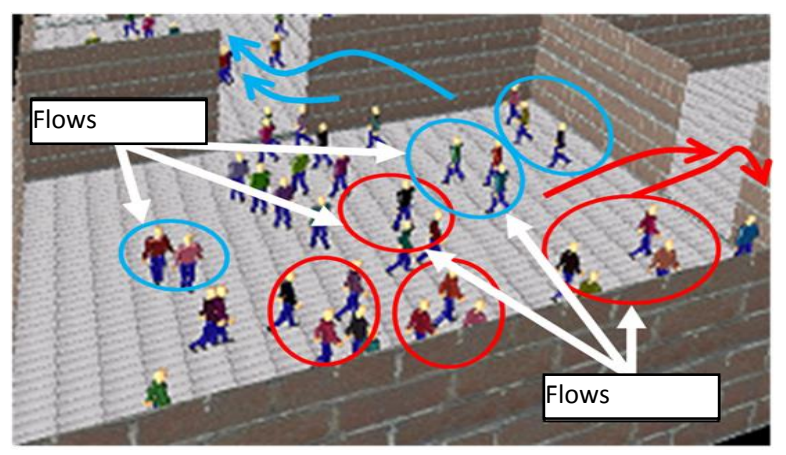

Fig. 7. Creation of small pedestrian flow in normal situation, these pedestrian flows are formed when the pedestrian density is high by using the macroscopic model

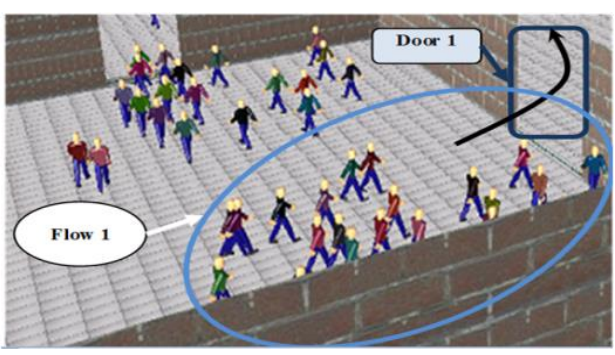

(a)

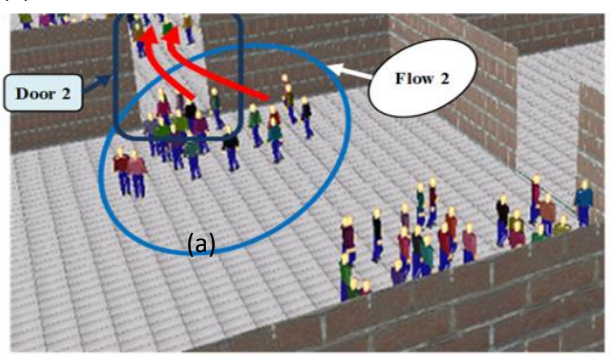

(b)

Fig. 8. Fig. 1. The small groups follow themselves for forming a flow

\section{CONCLUSION}

Multi-approach modeling is proposed in this work as an adaptive simulation strategy for exhibiting the pedestrian crowd movements and its emergent behaviors in high density situation. Our method makes it possible to exploit advantages from both macroscopic and microscopic models. The two types of models work simultaneously in a single simulation system, and are executed over different mutually exclusive partitions.

It is important to notice that our resulting hybrid technique can automatically and dynamically select the suitable strategy; the dynamic switching between both models is ruled by the runtime simulation metric which is the crowd density in the partitions of the virtual environment. Our model also ensures that no visible disturbance is generated when adaptively change the simulation method in a region. The partitioning of the environment allows us to define transition zones where the two types of movement modeling approach must be interacted and crowd under one regime must be moved to the other.

We will continue to work on the proposed behavior model, which aims to be useful in different kinds of crowd simulation applications, our future work provides a development of a wide variety of social behaviors. These behaviors are managed for more accurate simulation results under various complex conditions by incorporating the more complex group structures and the interactions between the different types of pedestrians. Further work, the coupling of mesoscopic models with our model will develop to apply in the region with middle density.

\section{REFERENCES}

[1] A. Kirchner, A. Shadschneider, "Simulation of evacuation processes using a bionics-inspired cellular automaton model for pedestrian dynamics". Physica A: Statistical Mechanics and its Applications, vol. 312(1), pp. 260-276, 2002 
[2] A. Treuille, S. Cooper, Z. Popovic, "Continuum crowds". ACM Transactions on Graphics (TOG), vol. 25(3), pp. 1160-1168, 2006

[3] A. Sud, R. Gayle, E. Andersen, S. Guy, M. C. Lin, D. Manocha, "Realtime navigation of independent agents using adaptive roadmaps", In ACM Symposium on Virtual Reality Software and Technology, Newport Beach, California, USA; pp. 99-106, 2007

[4] A. Sud, E. Anderson, S. Curtis, M. C. Lin, D. Manocha, "Real-time path planning in dynamic virtual environments using multiagent navigation graphs". Visualization and Computer Graphics, IEEE Transactions on, vol. 14(3), pp. 526-538, 2008

[5] A. Schadschneider, W. Klingsch, H. Klüpfel, T. Kretz, C. Rogsch, A. Seyfried, "Evacuation dynamics: Empirical results, modeling and applications". In: Encyclopedia of complexity and systems science. Springer New York, pp. 3142-3176, 2009

[6] B. Yersin, J. Maïm, F. Morini, D. Thalmann, (2008): Real-time crowd motion planning: scalable avoidance and group behavior. The Visual Computer: International Journal of Computer Graphics, vol. 24(10), pp. 859-870, 2008

[7] C.W. Reynolds, "Flocks, herds, and schools: A distributed behavioural model". In: Computer Graphics Proceedings of SIGGRAPH 1897, vol. 21(4), pp. 25-34, 1987

[8] C. Reynolds, "Steering behaviours for autonomous characters". In the proceedings of Game Developers Conference, San Francisco, California, pp. 763-782, 1999.

[9] D. Helbing, I. Farkas, T. Vicsek, "Simulating dynamical features of escape panic". Nature, vol. 407(6803), pp. 487-490, 2000

[10] D. Thalmann, S. R. Musse, "Crowd Simulation", 1st edn. Springer, 2007.

[11] F. Lamarche, S. Donikian, "Crowd of virtual humans: a new approach for real time navigation in complex and structured environments". In Computer Graphics Forum. Blackwell Publishing, vol. 23(3), pp. 509518, 2004

[12] F. Durupinar, J. Allbeck, N. Pelechano, N., Badler, "Creating crowd variation with the OCEAN personality model". In: Proceedings of the 7th International Joint Conference on Autonomous Agents and Multiagent Systems, Estoril, Portugal, 2008

[13] G. A. Kaminka, N. Fridman, "A cognitive model of crowd behavior based on social comparison theory". In Proceedings of the AAAI-2006 Workshop on Cognitive Modeling, Boston, USA, 2006

[14] H. Jiang, W. Xu, T. Mao, C. Li, S. Xia, Z. Wang, "Continuum Crowd Simulation in Complex Environments". Computers \& Graphics, vol. 34(5), pp. 537-544, 2010

[15] J. Pettré, J. P. Laumond, D. Thalmann, "A navigation graph for real-time crowd animation on multilayered and uneven terrain", In First International Workshop on Crowd Simulation, vol. 43(44), New York: Pergamon Press, pp. 194, 2005

[16] J. Van den Berg, S. Patil, J. Seawall, D. Manocha, M. C. Lin, "Interactive navigation of individual agents in crowded environments", In: Proceedings of the 2008 symposium on Interactive 3D graphics and games, ACM, Redwood Shores, CA, USA, pp. 139-147, 2008

[17] J. Sewall, D. Wilkie, M. C. Lin, "Interactive hybrid simulation of largescale traffic", ACM Transactions on Graphics, vol. 30 (6) , 2011

[18] L. Huang, S. Wong, M. Zhang, C. W. Shu, W.H. Lam, "Revisiting Hughes' dynamic continuum model for pedestrian flow and the development of an efficient solution algorithm", Transportation Research Part B: Methodological, vol. 43(1), pp. 127-141, 2009

[19] M. Sung, M. Gleicher, S. Chenney, "Scalable behaviors for crowd simulation". In: Computer Graphics Forum. Blackwell Publishing, vol.23(3), pp. 519-528, 2004

[20] M. Xiong, W. Cai, S. Zhou, M. Y. H. Low, F. Tian, D. Chen, D. W. S. Ong, B. D. Hamilton, "A case study of multi-resolution modeling for crowd simulation", In: Proceedings of the 2009 Spring Simulation Multiconference (SpringSim 2009), San Diego, California, USA, pp. 17, 2009

[21] M. Xiong, M. Lees, W. Cai, S. Zhou, M. Y. H. Low, "Hybrid modelling of crowd simulation". Procedia Computer Science, vol. 1 (1), pp. 57-65, 2010.

[22] M. Xiong, S. Tang, D. Zhao, "A hybrid model for simulating crowd evacuation". New Generation Computing, vol. 31(3), pp. 211-235, 2014

[23] M. L. Xu, H. Jiang, X. G. Jin, Z. Zhigang Deng, "Crowd simulation and its applications: Recent advances". Journal of Computer Science and Technology, vol. 29 (5), pp. 799-81, 2014

[24] N. Pelechano, J. Allbeck, N. I. Badler, "Controlling individual agents in high-density crowd simulation". In: Proceedings of the 2007 ACM SIGGRAPH/Eurographics Symposium on Computer Animation Eurographics Association, San Diego, CA, USA, pp. 108-118, 2007

[25] N. Pelechano, J. M. Allbeck, N. I. Badler, "Virtual Crowds: Methods, Simulation and Control". Synthesis Lectures on Computer Graphics and Animation, vol. 3(1), pp. 1-176, 2008

[26] N. Anh, Z. Daniel, N. Du, "A hybrid macro-micro pedestrians evacuation model to speed up simulation in road networks", Advanced Agent Technology, vol. 7068, pp. 371-383, 2012.

[27] R. Chighoub, F. Cherif, “',Multilayered framework for crowd microscopic simulation", International Conference on Multimedia Computing and Systems, 10-12 May 2012, pp. 366 - 371

[28] R. L. Hughes, "A continuum theory for the flow of pedestrians", Transportation Research Part B: Methodological, Vol. 36(6), pp. 507535, 2002

[29] R. Gayle, A. Sud, M. C. Lin, D. Manocha, "Reactive deformation roadmaps: motion planning of multiple robots in dynamic environments", In Intelligent Robots and Systems, IROS 2007. IEEE/RSJ International Conference, San Diego, California, USA, pp. 3777-3783, 2007.

[30] R. Y. Guo, H. J. Huang, "A mobile lattice gas model for simulating pedestrian evacuation". Physica A: Statistical Mechanics and its Applications, vol. 387(2), pp. 580-586, 2008.

[31] R. Narain, A. Golas, S. Curtis, M. C. Lin, "Aggregate dynamics for dense crowd simulation". ACM Transactions on Graphics (TOG) Proceedings of ACM SIGGRAPH, vol. 28(5), Asia, pp. 122, 2009

[32] S. R. Musse, D. Thalmann, "Hierarchical model for real time simulation of virtual human crowds", Visualization and Computer Graphics, EEE Transactions on, vol. 7(2), pp. 152-164, 2001

[33] S. Paris, S. N. Donikian, N. Bonvalet, "Environmental abstraction and path planning techniques for realistic crowd simulation". Computer Animation and Virtual Worlds, vol. 17(3), pp. 325-335, 2006

[34] S. Guy, J. Chuggani, S. Curtis, P. Dubey, M. Lin, D. Manocha, "Pledestrians: A least-effort approach to crowd simulation". In: Proceedings of the 2010 ACM SIGGRAPH/Eurographics symposium on computer animation. Eurographics Association, Madrid, Spain, pp. 119128,2010

[35] W. Shao, D. Terzopoulos, “Autonomous pedestrians”. In: Proceedings of ACM SIGGRAPH / Eurographics Symposium on Computer Animation, Los Angeles, USA, pp. 19-28, 2005

[36] W. Wang, Z. Lv, X. Li, W. Xu, B. Zhang, X. Zhang. "Virtual Reality Based GIS Analysis Platform”, Neural Information Processing. Springer International Publishing, vol. 9490, pp. 638-645, 2015.

[37] X. Zheng, T. Zhong, M. Liu, "Modeling crowd evacuation of a building based on seven methodological approaches", Building and Environment, vol. 44 (3), pp. 437-445, 2009

[38] Y. Q. Jiang, R. X. Liu , Y. L. Duan, "Numerical simulation of pedestrian flow past a circular obstruction", Acta Mechanica Sinica, vol. 27(2), pp. 215-221, 2011

[39] Y. Jiang, S. C. Wong, P. Zhang, R. Liu, Y. Duan, K. Choi, "Numerical simulation of a continuum model for bi-directional pedestrian flow", Applied Mathematics and Computation, Vol. 218 (10), pp. 6135-6143, 2012.

[40] Y. Jiang, Z. Peng. "Modeling and simulation of pedestrian flow through hydrodynamics", Procedia Engineering, International Conference on Advances in Computational Modeling and Simulation, vol. 31, 2012, pp. 1039-1044 